ISSN 0103-5150

Fisioter. Mov., Curitiba, v. 26, n. 2, p. 455-462, abr./jun. 2013

Licenciado sob uma Licença Creative Commons

\title{
0 ambiente virtual como interface na reabilitação pós-AVE: relato de caso ${ }^{1}$
}

\author{
Virtual environment as an interface device in \\ post-stroke rehabilitation: case report
}

\author{
Silvia Leticia Pavão ${ }^{[a]}$, Nara Vanessa da Costa Sousa ${ }^{[b]}$, Cinthia Monteiro Oliveira ${ }^{[b]}$, \\ Pedro Cláudio Gonsales de Castro ${ }^{[c]}$, Maria Cecília Moreira dos Santos ${ }^{[\mathrm{d}]}$
}

[a] Fisioterapeuta, mestre em Processos de Avaliação e Intervenção em Fisioterapia pela Universidade Federal de São Carlos (UFSCar), especialista em Reabilitação do Portador de Deficiência Física Incapacitante pelo Instituto de Medicina Física e Reabilitação do Hospital das Clínicas da Faculdade de Medicina da Universidade de São Paulo (USP), SP - Brasil, e-mail: silvia_pavao@hotmail.com

[b] Fisioterapeuta, especialista em Reabilitação do Portador de Deficiência Física Incapacitante pelo Instituto de Medicina Física e Reabilitação do Hospital das Clínicas da Faculdade de Medicina da Universidade de São Paulo (USP), São Paulo, SP - Brasil, e-mail: narinha.sousa@hotmail.com, ci.perdida@gmail.com

[c] Fisioterapeuta, mestre em Bioengenharia pelo Instituto de Medicina Física e Reabilitação do Hospital das Clínicas da Faculdade de Medicina da Universidade de São Paulo (USP), São Paulo, SP - Brasil, e-mail: pedroccastrofisio@hotmail.com

[d] Fisioterapeuta, mestre em Distúrbios do Desenvolvimento pelo Instituto de Medicina Física e Reabilitação do Hospital das Clínicas da Faculdade de Medicina da Universidade de São Paulo (USP), São Paulo, SP - Brasil, e-mail: mceciliamoreira@ig.com.br

\section{Resumo}

Introdução: 0 acidente vascular encefálico (AVE) é um quadro neurológico agudo de repercussões amplas, que exige de suas vítimas programas de reabilitação desafiadores na promoção da função. Nesse contexto, a Realidade Virtual (RV) é uma ferramenta de interface na reabilitação que pode gerar altos índices de motivação do paciente e permitir adaptação da terapia ao seu nível de função. Objetivo: Verificar o efeito da RV por

\footnotetext{
1 Apresentação em pôster no Congresso Brasileiro de Comportamento Motor, aprovação pela Comissão de Ética para Análise de Projetos de Pesquisa (CAPPesq) da Diretoria Clínica dos Hospital das Clínicas e da Faculdade de Medicina da Universidade de São Paulo, protocolo de pesquisa n. 0735/10.
} 
meio de videogame sobre o controle postural de um indivíduo pós-AVE. Materiais e métodos: Um indivíduo pós-AVE com um ano de lesão foi submetido a um protocolo de reabilitação física com videogame numa frequência de três vezes por semana por um período de 12 semanas. Anteriormente e após o programa foi realizada dinamometria por plataforma de força para análise de variáveis relacionadas ao centro de pressão (COP). Resultados: Na reavaliação, observou-se que a amplitude de deslocamento médio-lateral $(x)$ aumentou $67 \%$ na condição de olhos abertos (OA) e fechados (OF); amplitude anteroposterior $(y)$ aumentou 25 e $44 \%$ em OA e OF, respectivamente; área aumentou 109 e 141\% em OA e OF; velocidade diminuiu 26 e 0,27\% em OA e OF. Conclusão: A RV como interface na reabilitação possivelmente ampliou a exploração da base de suporte para manutenção da estabilidade, constituindo recurso adicional no tratamento desses indivíduos.

Palavras-chave: Acidente Vascular Cerebral. Equilíbrio postural. Jogos de vídeo. Biomecânica.

\section{Abstract}

Introduction: The impaired postural control is one of the main problems for stroke survivors. Its consequences require the development of effective and challenging rehabilitation strategies for promotion of function. In this context, studies focusing on Virtual Environment (VE) suggest that Virtual Reality (VR) is an interface device which allows creating high levels of motivation and adaptation of the patient to the therapy. Objective: The aim of this study was to investigate the role of VR using video game to the improvement of postural control in a chronic stroke survivor. Materials and methods: Only one subject participated in this study and was submitted to a rehabilitation protocol of 12 weeks, three times a week, using videogame. Measures of the center of pressure were obtained previously and latter to the protocol using a force plate. Results: The protocol resulted in higher amplitude of sway in $\mathrm{x}$ and $\mathrm{y}$ for both eyes open and eyes closed condition, and a higher area of sway in both conditions too. However, the velocity of sway was reduced for both conditions. Conclusion: The VR as an interface device in rehabilitation could be responsible for improve the exploration of the support base and thus might be an additional device in clinical rehabilitation.

Keywords: Stroke. Postural balance. Video games. Biomechanics.

\section{Introdução}

0 acidente vascular encefálico (AVE) é um quadro neurológico agudo, de origem vascular, que afeta a função cerebral com grande repercussão funcional (1). Dentre suas principais repercussões estão as disfunções de controle postural, que diminuem a independência do indivíduo (2).

A abrangência do comprometimento motor pós-AVE exige programas de reabilitação desafiadores ao paciente (3) a fim de trabalhar e restabelecer conexões cerebrais para promoção da função. 0 sucesso desses programas está vinculado à adesão do paciente (4).

No contexto de procura por técnicas efetivas e atraentes, a tecnologia vem ganhando importância no cenário de reabilitação. 0 uso da Realidade Virtual, experimentação de situações da vida real pelo sujeito por meio de um computador criando uma interface entre máquina e ser humano $(5,6)$, é exemplo dos avanços nesta área promovendo acesso a um ambiente multidimensional e multissensorial, $(5,7)$.

O uso da RV em reabilitação é embasado na ideia de que a execução de tarefas orientadas associada à observação de membros virtuais projetados na tela de um televisor possa facilitar a reorganização funcional dos sistemas motores e pré-motores afetados no AVE (8) e garantir a recuperação da função.

Aplicada à reabilitação, a Realidade Virtual permite adaptação da terapia ao grau de função do paciente, coleta de dados mais precisa para mensuração dos resultados obtidos com o protocolo aplicado e maiores índices de motivação com a reabilitação (9), fatores de grande influência sobre os resultados do tratamento.

O uso do videogame na reabilitação pós-AVE é um exemplo de uso da Realidade Virtual, associando tecnologia à interação com o paciente. Essa interface máquina/ser humano pode ocorrer por meio de videogames convencionais $(6,9)$, sistemas de captura 
do movimento por imagem (9), ou videogames interativos por acelerometria (10).

Uma das versões desses videogames interativos permite o rastreamento dos movimentos do indivíduo por meio do deslocamento do seu centro de gravidade sobre uma plataforma sensível à pressão (11). 0 acoplamento dos sinais da plataforma a uma televisão permite a reprodução de seus movimentos em ambiente virtual criando novas perspectivas ao processo de reabilitação, especialmente do controle postural $(7,9,12)$.

A literatura já evidenciou a eficácia da Realidade Virtual para a reabilitação de indivíduos com injúrias cerebrais; no entanto, a maioria dos estudos existentes nessa área tem foco na reabilitação da extremidade superior dos pacientes $(6,8,9)$ ou na função motora geral $(5,13)$, havendo pouca ênfase sobre a reabilitação postural e de membro inferior $(12,14)$.

Os estudos que avaliaram Realidade Virtual em reabilitação com uso de videogames interativos comparado à reabilitação convencional verificaram aumentos significativos na velocidade de marcha e no equilíbrio em ambos os grupos (12), ganhos adicionais na combinação dessas terapias, resultando em independência e função (14). No entanto, ainda são poucos os estudos envolvendo reabilitação do controle postural $(6,7,12,14)$.

Diante das vantagens do uso da Realidade Virtual e dos escassos estudos com videogame em reabilitação postural, supõe-se que o seu uso na reabilitação de indivíduos hemiparéticos possa promover ganhos no controle postural desses indivíduos.

Dessa forma, o objetivo do presente estudo foi o de verificar os efeitos de um protocolo de reabilitação utilizando Realidade Virtual baseado em jogos em ambiente virtual sobre o controle postural de um indivíduo pós-AVE.

\section{Métodos}

\section{Participante}

Participou do estudo um indivíduo do sexo masculino, de 46 anos, com lesão cerebral decorrente de AVE há um ano, inserido em um programa de reabilitação há sete meses com duas sessões semanais de fisioterapia. Quando admitido no estudo, o sujeito apresentava deambulação funcional com grau de espasticidade II em membros inferiores pela Escala modificada de Ashworth.

Para participar do estudo, ele assinou o Termo de Consentimento Livre e Esclarecido (TCLE) e foi informado de que seria descontinuado caso apresentasse mais de duas faltas consecutivas no protocolo proposto, ou nas sessões convencionais de fisioterapia.

\section{Procedimentos de teste}

Após aderir ao estudo, aprovado pelo Comitê de Ética em Pesquisa com Seres Humanos da Faculdade de Medicina da Universidade de São Paulo, parecer n. 0735/10, para a obtenção de dados antropométricos e determinação do nível de espasticidade, o indivíduo foi submetido a uma avaliação física no laboratório de análise do movimento do centro de reabilitação em que realizava seu tratamento.

Em seguida, foi realizada uma avaliação por meio de dinamometria em plataforma de força (15). Nessa avaliação, o indivíduo foi posicionado sobre a plataforma de força, em postura ereta, braços ao longo do corpo e pés paralelos na linha dos quadris. Foram realizadas seis tentativas de permanência estática por um período de tempo de 60 segundos cada uma, separadas por um descanso de 30 segundos (16, 17). Três tentativas ocorreram na condição de olhos abertos (OA) e três na de olhos fechados (OF) (15). Foi utilizada para análise apenas a terceira tentativa de cada condição, sendo as duas anteriores utilizadas como treino.

\section{Protocolo}

Após a avaliação inicial, o indivíduo foi submetido a um programa de treinamento com o videogame interativo, comercialmente conhecido por Nintendo Wii Fit, composto por um console acoplado a uma plataforma sensível a pressão e um controle remoto. Foram realizadas sessões terapêuticas em uma frequência semanal de três vezes, com duração de 30 minutos (18), ao longo de 12 semanas. Em cada sessão, eram propostos dois jogos diferentes, cada um deles era executado durante 15 minutos.

Foi utilizado o CD de jogos que acompanha o equipamento Wii Fit Plus com quatro opções de atividades: equilíbrio, ioga, treino de força e exercícios aeróbicos (Nintendo, Operations Manual wii 
fit). Para o presente estudo, foram escolhidas as atividades de equilíbrio.

Para a realização das atividades, o paciente posicionava-se sobre a plataforma de forças do videogame, com os pés paralelos a uma distância de 2,10 m do televisor. Em posição bípede, deveria cumprir as atividades propostas pelo equipamento, deslocando seu centro de gravidade sobre a plataforma de acordo com os objetivos dos jogos.

Foram utilizados os seis jogos de equilíbrio fornecidos pelo CD do videogame, previamente analisados pelos pesquisadores para determinação de seu nível de dificuldade. Dessa forma, a sequência de jogos escolhida para o protocolo ocorreu dos jogos mais simples para os de maior complexidade.

Além do protocolo, o indivíduo foi submetido à reabilitação convencional duas vezes na semana, com cinesioterapia, treino de equilíbrio e uso de Estimulação Elétrica Funcional em dorsiflexores do tornozelo; todos esses procedimentos eram realizados com o paciente desde sua inserção no programa de fisioterapia. Tomou-se o cuidado de manter, durante a realização do protocolo de intervenção com Realidade Virtual, os mesmos exercícios realizados na terapia convencional antes dessa intervenção para assegurar que as possíveis mudanças encontradas após 12 semanas fossem decorrentes do contato com o ambiente virtual.

Decorridas as 12 semanas de tratamento, o indivíduo foi reavaliado da mesma maneira descrita inicialmente.

\section{Análise dos dados}

A plataforma de força AMTI OR6-7, versão 2.0/2004, modelo OR6-7(1000), foi utilizada para determinação do comportamento do centro de pressão (COP) na postura estática em pé. 0 software EVArT foi usado para processamento dos dados.

A frequência de amostragem na aquisição dos dados foi de $100 \mathrm{~Hz}$, com posterior utilização de filtro Butterworth passa-baixa de segunda ordem, criticamente amortecido, com frequência de corte de 1050 Hz. O processamento dos dados da plataforma foi realizado pelo software EVArT.

Para análise do comportamento do COP foram eleitas as seguintes variáveis: amplitude de deslocamento de $x(\operatorname{Ampl} x)$, para o deslocamento médio-lateral; e de $y$ (Ampl $y$ ) para o deslocamento anteroposterior, área de deslocamento (Área) (19) e velocidade média do deslocamento do COP (Vel), calculada juntamente para $x$ e $y$ (20). 0 cálculo das variáveis nas coordenadas $x$ (COP $x$ ) e $y$ (COP $y$ ) no intervalo de 60 segundos foi realizado no software Excel. Foram eliminados os primeiros dez segundos de captação para cada uma das condições OA e OF por serem considerados períodos de adaptação à postura estática (15).

A análise dos dados foi feita por comparação entre os resultados das avaliações anterior e posterior ao protocolo para verificação do percentual de alterações ocorridas.

\section{Resultados}

A comparação entre os valores iniciais e finais das variáveis permitiu verificar a presença de alterações com o protocolo aplicado. Os valores absolutos de cada uma das variáveis podem ser vistos na Tabela 1.

Em relação à Ampl $x$, na condição $O A$ com a reavaliação pela plataforma de força, foi observado um aumento de $67,05 \%$ na amplitude de deslocamento médio-lateral. Para a condição OF, o aumento observado em $x$ após o protocolo foi de $67,51 \%$.

Em relação à Ampl $y$, para a condição $\mathrm{OA}$, foi constatado um aumento de $25,23 \%$ após o protocolo. $\mathrm{Na}$ condição OF, o aumento observado foi de 44,44\%.

Para a variável Área, na condição OA, na reavaliação, foi observado um aumento na área do CP de 109,22\%. Na condição OF o aumento observado para essa variável foi de 141,96\%.

Para Vel, na condição $\mathrm{OA}$, a reavaliação pela plataforma de força revelou uma diminuição da velocidade de 26,05\%. Na condição de OF, após o protocolo de intervenção foi constatado um aumento de $0,27 \%$ para esta variável.

\section{Discussão}

Os resultados do presente estudo fornecem subsídios de que o protocolo de intervenção terapêutica por meio de Realidade Virtual possivelmente exerceu influência sobre o comportamento do COP na postura estática em pé.

As amplitudes de deslocamento do COP tanto em $x$, quanto em $y$, aumentaram para as duas condições avaliadas, OA e OF; como consequência, a área de 
Tabela 1 - Desempenho relacionado ao comportamento do centro de pressão nas condições de olhos abertos e olhos fechados

\begin{tabular}{lcccc}
\hline Variável & \multicolumn{2}{c}{ Condição OA } & \multicolumn{2}{c}{ Condição OF } \\
\hline & Pré-Interv & Pós-Interv & 15,59 & Pós-Interv \\
Ampl $x(\mathrm{~mm})$ & 10,56 & 17,64 & 36,23 & 27,79 \\
Ampl $y(\mathrm{~mm})$ & 22,08 & 27,65 & 601,055 & 52,33 \\
Área $\left(\mathrm{mm}^{2}\right)$ & 233,164 & 487,746 & 36,42 & 1454,251 \\
Vel $(\mathrm{mm} / \mathrm{s})$ & 40,35 & 29,82 & 36,59 \\
\hline
\end{tabular}

Legenda: Ampl $x=$ amplitude de deslocamento de $x$; Ampl $y=$ amplitude de deslocamento de $y$; Área = área de oscilação; Vel = velocidade de oscilação; Pré-Interv = pré-intervenção; Pós-Interv = pós-intervenção; $0 A$ = olhos abertos; OF = olhos fechados.

Fonte: Dados da pesquisa.

oscilação também apresentou um aumento em ambas as condições; a velocidade de oscilação diminuiu após o protocolo aplicado. Entretanto, as considerações decorrentes desses resultados devem ser analisadas com cautela.

A maioria dos estudos sobre controle postural relaciona o aumento da oscilação do COP à menor estabilidade corporal $(21,22)$. No entanto, em indivíduos hemiparético, a lesão central acarreta deficit nas vias aferentes de regulação do controle postural. Assim, o aumento da oscilação pode ser uma estratégia de aumento do feedback proprioceptivo da região do tornozelo para compensar o deficit sensorial central (23).

Nesse sentido, a demanda corporal imposta pela Realidade Virtual por meio do videogame parece ter fornecido ao indivíduo estímulos para ampliar seus movimentos sobre a base de suporte, auxiliando-o em uma melhor exploração dessa base e aumentando as aferências proprioceptivas para manter a estabilidade. Essa maior exploração da base de suporte pode ser interpretada como uma adaptação funcional para melhor controle do corpo no espaço (24), pois em indivíduos pós-AVE a área de estabilidade encontra-se reduzida de 16 para 2,3\% (25).

Como observado, a Ampl $x$ (médio-lateral), na condição $\mathrm{OA}$, aumentou percentualmente mais que a Ampl y (anteroposterior) na reavaliação. Essa maior oscilação médio-lateral observada pode ser uma forma de compensação do deficit verificado no uso da estratégia de tornozelo para manutenção do equilíbrio. Indivíduos com lesões centrais apresentam menores índices de ativação de dorsiflexores de tornozelo, o que dificulta a utilização dessa estratégia (26). Oscilar médio-lateralmente é uma forma de evitar o uso dessa musculatura, com deficit de ativação após a lesão, para garantir a estabilidade corporal. Na condição OF, a oscilação observada em $x$ foi ainda maior porque com a privação da visão e de suas aferências para modulação postural, a quantidade de inputs somatossensoriais para os centros superiores do indivíduo deve aumentar (25, 27) para produzir feedback suficiente à manutenção da estabilidade que compense a ausência de visão. Essa maior oscilação pode ser uma adaptação funcional à atividade.

A interação com o ambiente virtual proporcionada pela Realidade Virtual fornecia feedback visual ao indivíduo; tanto pela informação do posicionamento do COP no televisor (28), quanto pela movimentação do personagem virtual na tela simulando a posição do indivíduo no espaço a partir da sua descarga de peso na plataforma. Esse feedback tem se mostrado eficiente na reabilitação postural e de percepção do corpo em indivíduos pós-AVE (29). Os treinos utilizando a Realidade Virtual em ambiente virtual podem ter extrapolado as condições reais de vida do indivíduo, auxiliando-o em estratégias de manutenção da estabilidade. No entanto, faltam ainda estudos que abordem a eficácia do uso de feedback visual acoplada à interatividade da Realidade Virtual.

Quanto à velocidade de oscilação do COP, considerada a variável mais fidedigna e reprodutível das relacionadas ao COP (20), observou-se diminuição percentual na reavaliação para condição $\mathrm{OA}$ e aumento para $\mathrm{OF}$.

Pode-se inferir que a redução de velocidade de oscilação do COP indica maior controle do indivíduo sobre 
sua estabilidade e alinhamento postural em posturas antigravitárias. Manter-se estável na vertical requer uma atuação conjunta da musculatura de membros inferiores e tronco com um padrão de ativação distal-proximal para geração de respostas posturais satisfatórias (23), respostas que geram oscilações do COP sobre a base de suporte (30). Portanto, a redução da velocidade, mesmo na presença de maiores amplitudes de oscilação pode indicar um maior controle sobre os padrões de ativação muscular, com aprimoramento das estratégias de manutenção do equilíbrio.

Na condição OF, observou-se um aumento da área e da velocidade de oscilação. Respostas assim são esperadas porque com a privação da visão, os inputs proprioceptivos tornam-se os principais contribuintes para modulação postural, requerendo maior utilização da estratégia de tornozelo, comprometida pelo baixo índice de ativação de dorsiflexores em hemiparéticos (25). Nessas condições são selecionadas estratégias alternativas de manutenção do equilíbrio, como as de quadril (30) que exigem maiores deslocamentos médio-lateral do COP, produzindo maiores índices de oscilação corporal.

Dessa forma, a menor estabilidade inerente à ausência da visão aumenta a oscilação corporal do indivíduo para gerar aferências proprioceptivas que lhe permitam manter-se equilibrado e sem cair (23). No entanto, ainda que a exploração da base de suporte seja maior nessa condição após o protocolo, o controle dessa oscilação foi menor, o que pode ser comprovado pelo aumento da velocidade de oscilação.

Pode-se observar, portanto, uma oscilação mais ampla e lenta no indivíduo do presente estudo, o que potencialmente é resultado de uma reorganização de suas estratégias posturais remanescentes para maior exploração de sua base de suporte, fatores importantes para a manutenção da estabilidade em tarefas da rotina diária com menores chances de desequilíbrio e queda.

Dessa forma, o uso da Realidade Virtual na reabilitação do controle postural, embora aparentemente não melhore os níveis de estabilidade do indivíduo, parece ter resultados importantes sobre as estratégias funcionais por ele utilizadas para manutenção da estabilidade. Esses resultados são alcançados não somente pelos movimentos desafiadores requeridos pelos jogos, mas também pela motivação que esses equipamentos trazem ao paciente. Resultados como esse são importantes, pois ampliam as possibilidades para reabilitação.
No entanto, muito embora o uso da Realidade Virtual tenha um papel promissor em reabilitação, com potencial de promover função e gerar adaptações funcionais para o movimento $(5,7,10,12,13$, 14), ele ainda é pouco explorado na literatura e talvez ainda não faça da rotina da maioria dos sujeitos inseridos na reabilitação.

0 que se pode afirmar é que, embora os resultados como os apresentados no presente estudos sejam promissores, a Realidade Virtual não substitui absolutamente a terapia convencional e seu uso deve ser feito de forma criteriosa e contextualizada.

Vale ainda ressaltar que as inferências do presente estudo são limitadas pelo tamanho reduzido da amostra e ausência de um follow-up que tenha verificado a manutenção dos ganhos obtidos com a intervenção proposta. Para estudos futuros, pretende-se implantar grupos de intervenção com amostras maiores, grupo controle e follow-up, fatores que podem aumentar a abrangência dos resultados encontrados.

\section{Conclusão}

Pode-se concluir, portanto, que a implementação da Realidade Virtual em reabilitação permitiu ampliação e aprimoramento da exploração da base de suporte, sendo um recurso interessante para ser adicionado à terapia convencional.

\section{Referências}

1. Green J, Forster A, Young J. A test-retest reliability study of the Barthel Index, the Rivermead Mobility Index, the Nottingham extended Activities of Daily Living Scale and the Frenchay Activities Index in stroke patients. Disabil Rehabil. 2001;23(15):67076. doi:10.1080/09638280110045382.

2. Srivastava A, Taly AB, Gupta A, Kumar S, Murali T. Post stroke balance training: Role of force platform with visual feedback technique. J Neurol Sci. 2009;287:8993. doi:10.1016/j.jns.2009.08.051.

3. Winckel AV, Feys H, Lincoln N, Weerdt W. Assessment of arm function in stroke patients: Rivermead Motor Assessment arm section revised with Rasch analysis. Clin Rehabil. 2007;21:471-79. doi:10.1177/ 0269215507071783. 
4. Brewer BW, Van Raalte JL, Petitpas AJ, Sklar JH, Pohlman MH, Krushell RJ, et al. Preliminary psychometric evaluation of a measure of adherence to clinic-based sport injury rehabilitation. Phys Ther Sport. 2000;1:68-74. doi:10.1054/ptsp.2000.0019.

5. Wuang YP, Chiang CS, Su CY, Wang CC. Effectiveness of virtual reality using Wii gaming technology in children with Down Syndrome. Res Dev Disabil. 2011;32:31221. doi:10.1016/j.ridd.2010.10.002.

6. Halton J. Virtual rehabilitation with video-games: a new frontier for occupational Therapy. J. Occup Ther. 2008;9(6):12-14.

7. Deutsch JE, Borbely M, Filler J, Huhn K, GuarreraBowlby P. Use of a low-coast, comercially, avaible game console (wii) for rehabilitation of an adolescent wth cerebral palsy. Phys Ther. 2008;88(10):1196-1207. doi:10.2522/ptj.20080062.

8. Cameirão MS, Bermúdez I Badia S, Oller ED, Verschure PFMJ. Neurorehabilitation using the virtual reality based Rehabilitation Gaming System: methodology, design, psychometrics, usability and validation. J Neuroeng Rehabil. 2010;7(48):1-14.

9. Huber M, Rabin B, Docan C, Burdea G, Nwosu ME, Abdelbacy M, et al. Play-Station 3-Based tele-rehabilitation for children with hemiplegia. 2008;25-27:105-12.

10. Butler DP, Willett K. Wii rehabilitation: Is there a role in trauma? Injury, Int J. Care Injured. 2010;41:883-85.

11. Nintendo Corporate. Nintendo of America Inc. 2009; [cited Aug. 12nd 2009]. Available at: http://www. nintendo.com/corp.

12. Deutsch JE, Robbins E, Morrinson J, Bowlby PG. Wiibased compared to standard of care balance and mobility rehabilitation for two individuals post-stroke. 2009:117:20

13. Saposnik G, Mamdani M, Bayley M, Thorpe KE, Hall J, Cohen LG, et al. Effectiveness of virtual reality exercises in stroke rehabilitation (EVREST): Rationale, design and protocol of a pilot randomized clinical trial assessing the Wii game system. Int J Stroke. 2010;5:4751. doi:10.1111/j.1747-4949.2009.00404.x.

14. Kim JH, Jang SH, Kim CS, Jung LH, You JH. Use of virtual reality to enhance balance and ambulation in chronic stroke: a double-blind, randomized controlled study. Am J Phys Med Rehabil. 2009;88:693-701. doi:10.1097/PHM.0b013e3181b33350.
15. Freitas SMSF, Duarte M. Métodos de análise do controle postural. São Paulo: Laboratório de Biofísica; 2006.

16. Santos, AD, Degani AM, Zatsiorsky VM, Latash ML. Is Voluntary control of natural postural sway possible? J Mot Behav. 2008:40(3):79-185.

17. Noronha M, Refshauge KM, Kilbreath SL, Crosbie J. Loss of proprioception or motor control is not related to functional ankle instability: an observational study. Aust J Physiother. 2007;53:193-98. doi:10.1016/ S0004-9514(07)70027-2.

18. Chen C, Cheng PT, Chen CL, Chen SC, Chung CY, Yeh TH. Effects of Balance Training on Hemiplegic Stroke Patients. Chang Gung Med J. 2002;9(25):583-90.

19. Winter DA. Balance and posture in human gait. In: Winter DA, editor. A.B.C. (Anatomy, Biomechanics, and Control of balance during standing and walking). Waterloo, Ontario: Waterloo Biomechanics, University of Waterloo; 1995. p. 41-54.

20. Raymakers JA, Samson MM, Verhaar HJ. The assessment of body sway and the choice of stability parameter(s). Gait Posture. 2005;21:48-58. doi: 10.1016/j.gaitpost.2003.11.006.

21. Hyndman D, Pickering RM, Ashburn A. Reduced sway during dual task balance performance among people with stroke at 6 and 12 months after discharge from hospital. Neurorehabil and Neural Repair. 2009;23:847-54. doi:10.1177/1545968309338192.

22. Hasan SS, Lichtenstein MJ, Shiavi RG. Effect of loss of balance on biomechanics platform measures of sways: measures of stance and a method for adjustment. J. Biomech. 1990;8(23):783-89. doi:10.1016/ 0021-9290(90)90025-X.

23. Patla A, Frank J, Winter D. Assessment of balance control in the elderly: major issues. Phisiotherapy Canada. 1990;42(2):89-97. doi:10.3138/ptc.42.2.089.

24. Duarte M. Análise estabilográfica da postura ereta humana quasi-estática. [Tese] São Paulo: Universidade de São Paulo; 2000.

25. Geurts ACH, Haart M, Van Nes IJW, Duysens J. A review of standing balance recovery from stroke. Gait Posture. 2005;22(3):267-81. doi:10.1016/j.gaitpost. 2004.10.002. 
26. Woollacott MH, Shumway-Cook A. Postural dysfunction during standing and walking children with cerebral palsy: What are the urderlying problems and what new therapies might improve balance? Neural Plast. 2005;12(2-3):211-19. doi:10.1155/NP.2005.211.

27. Forssberg H, Nashner LM. Ontogenetic development of postural control in man: Adaptation to altered support and visual condition during stance. 1982;2:545-52.

28. Clark RA, Bryant AL, Pua Y, Mccrory P, Bennell K, Hunt M. Validity and reliability of the Nintendo Wii Balance Board for assessment of standing balance. Gait Posture. 2009;2920:1-4.
29. Shumway-Cook A, Anson D, Haller S. Postural sway biofeedback: its effect on re-establishing stance stability in hemiplegic patients. Arch Phys Med Rehabil. 1988;69:395-400. PMid:3377664.

30. Garland SJ, Gray VL, Knorr S. Muscle activation patterns and postural control following stroke. Motor Control. 2009;13:387-411.

Recebido: 28/04/2012

Received: 04/28/2012

Aprovado: $15 / 12 / 2012$

Approved: 12/15/2012 